by means of strain gauze.

The results were as follows:

1) Oral glycerol and isosorbide solutions effectively reduced intracranial pressure and produced osmotic diuresis mildly. Their effectiveness appeared 30 to 60 minutes after oral administration and remained over 2 to 3 hour period. There were no remarkable secondary rebound phenomena.

However, glycerol was more difficult than isosorbide to be given orally because of its bitterness.

2) In urea maximal effectiveness occurred 2 to 3 hours after oral administration and it remained over 2 to 3 hour period. Its action was stronger than one of the other two osmotic agents, but it produced secondary rebound overshoot and gastric irritation. (We found it very difficult to give urea per os.)

3) In 2 patients with subfrontal tumors and one patient with a pituitary tumor, long-continued administration of isosorbide produced changes in serum electrolytes such as hypernatremia followed by hypopotassium.

From above-described results, we concluded that long term administration of isosorbide was possible and produced few side effects. It is also useful not only in in-clinic patients, but also in out-clinic patients for its mild efficacy.

\title{
118. The Clinical Evaluation of Steroid injected into the Ventricular System
}

\author{
Shiro IMamura and Humiaki KaI \\ 1st Department of Surgery, Kumamoto University School of Medicine
}

Steroid hormone, usually administered intravenously or intramuscularly, has become popular for treatment of cerebral edema. In this experiment with mongrel dogs, predonine was administered intraventricularly and its concentration in the brain tissue were studied in comparison with those on intravenous administration.

(I) Measurement of predonine in the brain tissue.

Dogs were injected with predonine intravenously (through the cephalic vein), intraarterially (through the carotid artery), intraventricularly (into the lateral ventricle through a burr-hole) or intracisternally (into the cisterna magna percutaneously). The animals were sacrificed with thiopental-sodium at various time intervals after administration of predonine. One gram of cerebral tissue was taken from various sites of the brain (cortex and white matter of hemisphere, thalamus, pons, and cerebellum). From each of the specimens, dichloromethanesoluble substance was extracted.

The extract was treated according to Silber-Poter's method for color development. Twenty-one hours after color development, absorbance of the colored 
product was measured at the wavelength of $410 \mathrm{~m} \mu$ using Shimazu's M-P-S 50 spectrophotometer.

(II) Evaluation of effect to protect cerebral edema.

Fifteen minutes after dogs were injected with RISA $(50 \mu \mathrm{c} / \mathrm{kg})$ intravenously, predonine $(20 \mathrm{mg})$ was injected the animals intraventricularly or intravenously. Thirty minutes after predonine injection, cerebral edema was produced by injecting with vegetable oil $(0.1 \mathrm{cc} / \mathrm{kg})$ into the carotid artery. Thirty minutes after the oil injection, the brain tissue was obtained from the cortex in the parietal region, and its radioactive counts $(\mathrm{c} / \mathrm{m} / \mathrm{g})$ were measured by using the Aloka's TDC- 6 , well-type scintillation counter.

(III) Results.

The concentration of predonine in the brain tissue reached the maximum level $30 \mathrm{~min}$. after its intraventricular administration, being 30 times as high as that obtained by intravenous or intraarterial injection. The radioactivity of the brain tissue into which predonine was given by intraventicular injection was $9708 \mathrm{c} / \mathrm{m} / \mathrm{g}$, while the radioactivity was $38300 \mathrm{c} / \mathrm{m} / \mathrm{g}$, in the case of intravenous injection and $76503 \mathrm{c} / \mathrm{m} / \mathrm{g}$ in the case of the control run with non-treated dogs. (The radioactivity of the normal brain tissue was $4798 \mathrm{c} / \mathrm{m} / \mathrm{g}$ ).

\title{
119. Experimental Study on Acute Brain Swelling
}

\author{
Susumu Ishikawa, Hiromichi Hibino, Junji GoIshi and Kohji Mrtsuda \\ 2nd Department of Surgery, Hiroshima University School of Medicine
}

Evacuation of acute intracranial hematoma may be followed by rapidly developing brain swelling.

The present experiment was attempted to determine the sequence of events during gradual expansion of an intracranial mass lesion, and to find certain events which is predictable development of the acute brain swelling after prompt decompression.

Under light anesthesia with pentobarbital a balloon was expanded in the supra- or infratentrial epidural space of the dog, which were ventilated spontaneously. Respiration, systemic blood pressure, cisterna magna pressure, pressure in the sinus confluence, venous blood flow from the confluence, ECG, and EEG were continuously recorded. On the basis of alterations in systemic blood pressure and pulse rate during supratentrial compression, the following 5 stages were defined. Stage 0; no change in pulse and blood pressure. Stage 1; bradycardia and slight fall in blood pressure. Stage 2; bradycardia and rise in blood pressure. Stage 3; suddenly appearing tachycardia and rise in blood pressure. Stage 4; disappearance of tachycardia, bradycardia and fall in blood pressure. 